crystal device to present the different faces to the incident light.

This technique offers a new, potentially easier, and more effective approach for the fabrication of highly ordered photonic crystals than currently used. According to the researchers, further refinement of this method may yield even greater advantages by shortening fabrication time and further reducing defects.

KRISTA NIECE

\section{New Coating Method Produces Arrays of Orthogonally Tilted Nanopores in Ceramic Films}

Surfactant-templated thin mesoporous ceramic films with hexagonally closepacked (hcp) cylindrical nanopores aligned perpendicular to the substrate attract more interest than films with pores aligned parallel to the substrate surface in fields such as membrane separations, sensors, and catalysis, because their channels are accessible to larger solutes. V.R. Koganti and S.E. Rankin from the University of Kentucky, D. Dunphy of Sandia National Laboratories, V. Gowrishankar and M.D. McGehee from Stanford University, and X. Li and J. Wang from Argonne National Laboratory have demonstrated that a chemically neutral sacrificial copolymer layer directed the orientation of orthogonally tilted hcp pores in surfactant-templated silica and titania films.

As reported in the November 8, 2006, issue of Nano Letters (p.2567; DOI:10.1021/ nl061992v), chemically neutral layers were formed by coating clean glass substrates with PEO-r-PPO copolymer (PEO-poly (ethylene oxide); PPO-poly (propylene oxide)) and cross-linking the films by an isocyanate hydroxyl reaction at $120^{\circ} \mathrm{C}$ overnight. Then, a film of silica sol was dip-coated onto the substrates, with P123 (PEO-co-PPO-co-PEO) as a surfactant pore template and tetraethylorthosilicate as a silica precursor. The films were aged and dried at $40^{\circ} \mathrm{C}$ for $24 \mathrm{~h}$ and $100^{\circ} \mathrm{C}$ for $24 \mathrm{~h}$, and calcined in air at $500^{\circ} \mathrm{C}$. The researchers controlled the thickness of the silica films by diluting the original sol in ethanol. Similarly, surfactant-templated titania films were produced by dip-coating titania sols, prepared by adding a solution of P123 in ethanol to a mixture of titanium(IV) ethoxide and concentrated $\mathrm{HCl}$, onto PEO-r-PPO copolymer-modified surfaces. Titania films were aged in a highly humid environment at $4^{\circ} \mathrm{C}$ for $2 \mathrm{~h}$ and calcined at $400^{\circ} \mathrm{C}$.

$X$-ray diffraction and grazing incidence small-angle $x$-ray scattering patterns confirmed that the films contained hcp chan- nels tilted perpendicular to the substrate so long as the film thickness was below a critical value between $70 \mathrm{~nm}$ and $100 \mathrm{~nm}$. Thicker films (up to $240 \mathrm{~nm}$ thick) could be completely oriented by sandwiching them between two PEO-r-PPO-modified substrates. Direct observation by electron microscopy corroborated these results.

Because the cross-linked PEO-PPO layer masks substrate-specific effects, the researchers said that orthogonally tilted hcp nanopore ceramic films can be deposited on substrates of any curvature and size onto or into which the PEO-PPO layer can be deposited. Other ceramic precursors may allow extension of this approach to other applications, including photovoltaics and fuel cells, with the added advantage that this is a cheap and rapid procedure that can be easily incorporated into microelectronic fabrication processes.

JOAN J. CARVAJAL

\section{Nanoparticles Conjugated with Tobacco Mosaic Virus Form Electronic Memory Device}

The unique properties of nanostructured biomaterials make them ideal candidates for next-generation electronic nanodevices. Although electrical switching has been observed for nanoparticles incorporated into organic polymer thin films, electronic devices fabricated directly from biomaterials are largely unsuccessful due to a lack of charge transport. Recently, however, Y. Yang, C.S. Ozkan, and coresearchers from the University of California, Los Angeles, and the University of California, Riverside, have demonstrated an electronic memory effect in a device featuring inorganic-biomaterial composite nanowires.

As reported in an article recently published in the October 2006 issue of Nature Nanotechnology (p. 72; DOI: 10.1038/ nnano.2006.55), Yang, Ozkan, and coresearchers formed nanowires by incorporating platinum nanoparticles (Pt NPs) with tobacco mosaic virus (TMV), which is rigid and tubular in shape with a length of about $300 \mathrm{~nm}$, outer diameter of $18 \mathrm{~nm}$, and inner diameter of $4 \mathrm{~nm}$. Electroless deposition with a Pt-ion solution conjugated the NPs with TMV. Transmission electron microscopy and atomic force microscopy (AFM) showed that an average of about $16 \mathrm{Pt}$ NPs per virus, with an average diameter of $10 \mathrm{~nm}$, were evenly distributed over the TMV surface, although NP clustering was observed at each nanowire node. Photoelectron spectra showed that the primary binding site of the Pt NPs was the outer surface of TMV. The researchers followed a simple fabrication process: thermal evaporation was used to deposit bottom aluminum electrodes onto a glass substrate; a thin film of Pt-TMV nanowires in an insulating poly(vinyl alcohol) (PVA) matrix was added by spin-coating; sandwiching the film with electrodes deposited onto glass completed the device. The thicknesses of the electrodes and thin films were determined with a profilometer to be $70 \mathrm{~nm}$ and $60 \mathrm{~nm}$, respectively. The active area of the device was $0.04 \mathrm{~mm}^{2}$.

Yang, Ozkan, and co-researchers showed that the nonlinear current-voltage $(I-V)$ behavior exhibited by their device arises from bistable electrical states. An abrupt increase in current by more than three orders of magnitude (from $10^{-8} \mathrm{~A}$ to $10^{-5} \mathrm{~A}$ ) was observed once a threshold bias of $3.1 \mathrm{~V}$ was reached. The stability of this "on," or high-conductance, state was demonstrated by a second scan. During a reverse scan from 0 to $-5 \mathrm{~V}$, the on-state was switched to the off, or low-conductance, state-that is, from $10^{-5} \mathrm{~A}$ to $10^{-7} \mathrm{~A}$, at a turn-off bias of -2.4 V. Control devices, in which the PVA thin film contained either TMV or Pt NPs, did not display conductance switching.

From measurements of current and voltage as functions of temperature, the researchers deduced that the conduction mechanisms are thermionic injection in the off-state and charge tunneling in the on-state. Conductance AFM was also used to demonstrate conductance switching in a single TMV-Pt nanowire. The researchers programmed their device for repetitive write/read/erase cycles and found difficulty in returning to the offstate after about 400 cycles, which they believe was caused by degradation of the TMV-Pt interface due to Joule heating. An analysis of the temperature-dependence of the memory retention led the researchers to insights into the charge storage mechanism, which has implications on the potential of their device for commercialization. The researchers said that charge trap sites are located on the Pt NPs and that the TMV protein coating effectively acts as an energy barrier. The activation energy of the charge trap was calculated to be $0.15 \mathrm{eV}$, which the researchers noted is close to the charging energy of similarly sized NPs. Yang, Ozkan, and co-researchers said that the composition of the TMV core- - RNA with aromatic rings rich with electrons-can act as charge donors; the function of the TMV is therefore not merely to provide a backbone for the organization of the NPs. The researchers said that "the retention stability and cycling endurance of the device could be further improved by introducing smaller-sized or other types of nanoparticles" and that "the concept of 
conjugating nanoparticles with biomolecules opens up new possibilities for making functional electronic devices using biomaterial systems."

STEVEN TROHALAKI

\section{Germanium Nanocrystals Embedded in Glass Exhibit Large Melting-Point Hysteresis}

J.W. Ager III, Q. Xu, D.C. Chrzan, and E.E. Haller of Lawrence Berkeley National Laboratory (LBNL) and the University of California, Berkeley, P. Kluth of the Australian National University, and their colleagues have discovered that nanocrystals of germanium embedded in silica glass do not melt until the temperature rises almost $200 \mathrm{~K}$ above the melting temperature of germanium in bulk. These melted nanocrystals have to be cooled more than $200 \mathrm{~K}$ below the bulk melting point before they resolidify. Haller explains that beyond broad scientific interest, the properties of germanium nanoparticles embedded in amorphous silicon dioxide matrices have promising applications.

"Germanium nanocrystals in silica have the ability to accept charge and hold it stably for long periods, a property which can be used in improved computer memory systems. Moreover, germanium dioxide mixed with silicon dioxide offers particular advantages for forming optical fibers for long-distance communication."

To exploit these properties means understanding the melting/freezing transition of Ge under a variety of conditions. The researchers embedded nanoparticles averaging $2.5 \mathrm{~nm}$ in diameter in silica. As they reported in the October 13, 2006, issue of Physical Review Letters (155701; DOI: 10.1103/PhysRevLett.97.155701), the researchers made silica glass samples $500 \mathrm{~nm}$ thick by oxidizing pure silicon wafers in steam. They implanted germanium ions in the amorphous silica and then annealed the sample at $900^{\circ} \mathrm{C}$ to form nanocrystals. The transparent glass allowed characterization of the embedded nanocrystals by Raman spectroscopy. The glass was also readily etched away for examination of the nanocrystals with an atomic force microscope.

Heating and cooling of the samples were performed in situ in a transmission electron microscope. By thinning the sam- ples to less than $300 \mathrm{~nm}$, the researchers could observe the electron diffraction rings produced by the crystal lattices of the embedded particles. When the particles began to melt, the diffraction rings weakened and vanished, allowing precise measurement of the temperature at which the embedded particles melted. As the temperature was lowered again, the appearance of the diffraction rings signaled resolidification.

For most materials, interface energies between solid and vapor-for example, a bar of gold in air-favor the formation of a liquid surface layer as the temperature increases, which continues to grow until the entire object is melted; this liquid layer forms more readily at lower temperatures as the proportion of surface to volume increases. Haller notes that "if you make free-standing nanoparticles of gold small enough, they melt at room temperature."

Embedded nanocrystals occasionally behave differently, however. Superheating has been observed in the case of nanocrystals embedded in a crystalline matrix, for example, nanoparticles of lead embedded in an aluminum matrix. This is attributed to the lattice structures of the two crystals "locking up," suppressing the vibration of the nanoparticles' surface atoms that would lead to melting. But Ge nanocrystals in silica glass are a different matter: the glass matrix has no lattice structure to lock with the surface of the germanium crystal. Ager said that "because there was no lattice structure in the matrix, we had naively expected the germanium crystals to behave more like freestanding nanoparticles - that is, we expected the melting temperature to be much less than in bulk germanium. Instead, to our surprise, germanium nanocrystals in glass had to be superheated to melt."

That was only the first surprise. In bulk materials, the interface energy between solid and vapor, which allows the transition from solid to liquid at the melting temperature, creates a roadblock in the opposite direction, an energy barrier to freezing.

"It always costs energy to form a surface," said Chrzan. "In the bulk, in fact, it's possible to supercool many materials and maintain them in a liquid state well above their normal freezing/melting point. In order to freeze, a material must overcome that slight energy barrier so as to form a critical solid nucleus."

In the case of Ge nanocrystals embedded in glass, the same large interface energy barrier that leads to superheating before the solid crystal can melt means the melted inclusions must be supercooled before they freeze.

"While these results were unexpected," Chrzan said, "it turns out they can be explained in a straightforward way. We modified the traditional theory of nucleation developed by David Turnbull in the 1950s. Even though in our system, the ratio of surface to volume is far greater than in the bulk materials Turnbull was working with - and even though, instead of a solid-vapor interface, we are working with a solid-glass interface-we saw that we could apply his theory in this new regime."

Chrzan said, "Typically in bulk materials, surface premelting means there's no need for nucleation before melting occurs. But in our case, the large proportional surface area of the germanium nanoparticles, plus the interface energy of the solid-glass interface, creates a calculable nucleation barrier in both directions."

As the nanoparticle heats up, a liquid nucleus, its lens shape partly determined by the confining spherical cavity in the glass, must achieve a critical size before it can spread and entirely melt the nanocrystal. Conversely, as the temperature drops, a solid nucleus forms and starts to grow from the surface of the liquid sphere-a nucleus that will eventually cause the entire nanometer-sized liquid globule to freeze into a solid crystal. The Turnbull theory as modified by Chrzan predicted the temperatures at which both events would occur.

"Melting and freezing points for materials in bulk have been well understood for a long time," said Haller, "but whenever an embedded nanoparticle's melting point goes up instead of down, it requires an explanation. With our observations of germanium in amorphous silica and the application of a classical thermodynamic theory that successfully explains and predicts these observations, we've made a good start on a general explanation of what have until now been regarded as anomalous events."

\section{Missing Important Issues of MRS Bulletin?}

Back Issues are still available! Contact MRS for details-

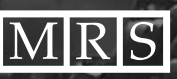

506 Keystone Drive, Warrendale, PA 15086-7573 U.S.A.

Tel: 724-779-3003 - Fax: 724-779-8313

E-mail: info@mrs.org·www.mrs.org/publications 